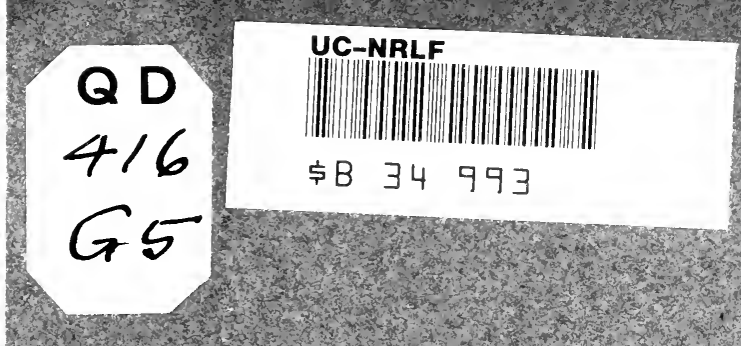




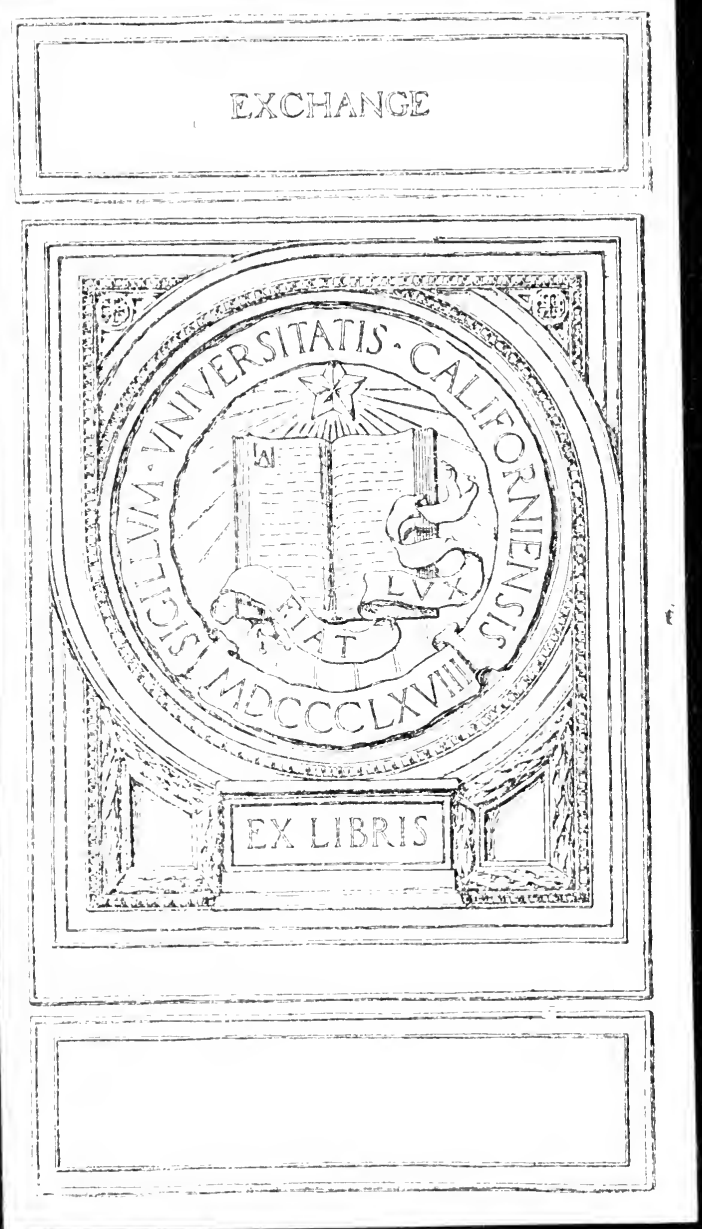




\title{
INVESTIGATION OF BROMONITROCAMPHANE
}

\author{
BY \\ PAUL, MEADE GINNINGS
}

B.S. University of Illinois, 1919

M.S. University of Illinois, 1920

THESIS

SUBMITTED IN PARTIAL FULFILIMMENT OF THE REQUIREMENT FOR THE DEGREE OF DOCTOR OF PHILOSOPHY IN CHEMISTRY IN THE GRADUATE SCHOOL, OF THE UNIVERSITY OF ILIINOIS, 1922.

[Reprinted from the Journal of the American Chemical Socintr, Vol. XLIV, No. 11. November, 1922.] 


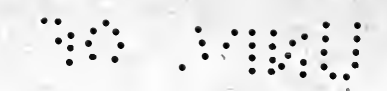

@ 


\title{
INVESTIGATION OF BROMONITROCAMPHANE
}

\author{
BY \\ PAUL MEADE GINNINGS \\ B.S. University of Illinois, 1919 \\ M.S. University of Illinois, 1920
}

THESIS

SUBMITTED IN PARTIAL FULFILLMENT OF THE REQUIREMENT FOR THE DEGREE OF DOCTOR OF PHII.OSOPHY IN CHEMISTRY IN THE GRADUATE, SCHOOI, OF THE UNIVERSITY OF ILLINOIS, 1922.

[Reprinted from the Journal of the Amenican Chemical Societr, Vol. XLIV, No. 11. November, 1922.] 


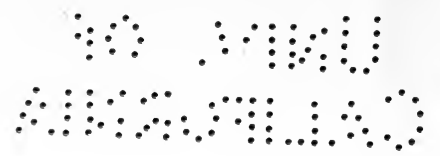

E. WIE 


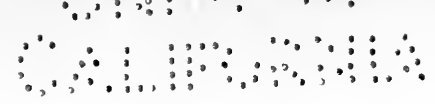

\section{ACKNOWLEDGMENT}

The author wishes to take this opportunity to express his indebtedness to Prof. W. A. Noyes, upon whose suggestion this investigation was undertaken, and under whose kind direction it was carried out. 


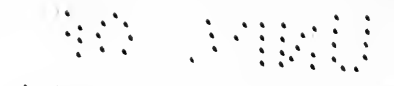

$\therefore \therefore \because \because \because \because \cdots$ 


\section{INVESTIGATION OF BROMONITROCAMPHANE ${ }^{1}$}

About 20 years ago Forster $^{1}$ discovered that when potassium hypobromite is allowed to act on camphoroxime, a bromonitro compound is obtained which he named bromonitrocamphane and to which he assigned the structure I.
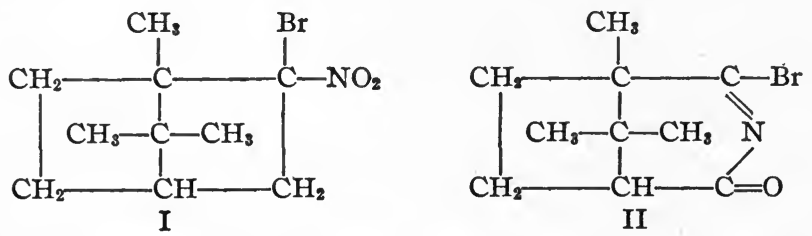

He and his collaborators established fairly conclusively the main points

1 Forster, J. Chem. Soc., 71, 199 (1897); 71, 1030 (1897); 75, 1141 (1899); 77, 251 (1900); 79, 108 (1901); 79, 264 (1901); 79, 644 (1901); Ref. 4; 79, 987 (1901); 79, 1003 (1901); 81, 865 (1902); 83, 78 (1903); Proc., 28, 313 (1912). 
of interest, but there were two things not accomplished. One is the oxidation of bromonitrocamphane and identification of the products, and the other, proof that the bromonitrocamphane anhydride (II) contained the carbonyl group in its molecule. In view of the above facts, it seemed desirable to investigate further the above compounds.

When bromonitrocamphane is treated with alcoholic silver nitrate, it loses the elements of hydrogen bromide and forms Compound III with the bridge or trimethylene ring in the molecule. This can be taken by several steps to an isomer of camphor which has the nitro group in III replaced by an hydroxy group.

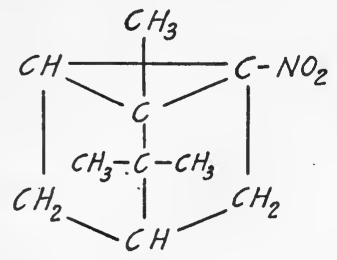

III

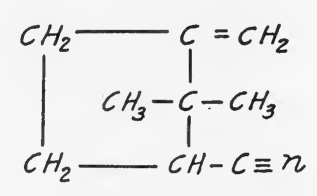

IV

When bromonitrocamphane is treated with conc. sulfuric acid in the cold, it loses the elements of water to form the anhydride, which is easily converted into an isomer. This anhydride and its isomer are changed very easily into an unsaturated nitrile (IV) and this nitrile can be hydrolyzed to the corresponding unsaturated acid, named infra-campholenic acid by Forster.

Oxidation of bromonitrocamphane was tried out some time ago ${ }^{2}$ but under the conditions of the oxidation, no camphoric acid or camphoronic acid was obtained. These should be the products of the oxidation if the bromine and nitro groups are situated in the $-\mathrm{CO}-\mathrm{CH}_{2}-$ side of the camphor molecule. The oxidation has, however, been repeated in this investigation under more varying conditions and camphor, camphoric acid, camphoronic acid isolated from the products of the oxidation.

The formation of bromonitrocamphane by the action of potassium hypobromite on camphoroxime is rather unusual. Evidently, the synthesis involves both bromination and oxidation. In his original work on this compound Forster thought that the mechanism of the reaction was bromination to bromonitrosocamphane, and then oxidation to the bromonitrocamphane. He noticed that a green compound was formed during the synthesis and he suggested that this was a hydrate of bromonitrocamphane, since it gave bromonitrocamphane on exposure to air, or on drying. The mechanism of the synthesis has been investigated in detail in this paper. The results indicate that the mechanism is partially as Forster thought,

${ }^{2}$ Gwinn, Thesis, University of Illinois, 1920. 
that is bromination and then oxidation, but that no hydrate of bromonitrocamphane is formed. It has been found that the green color is due to a product of a side reaction, hydroxy-nitrosocamphane. This is due to the fact that potassium hypobromite can act either as an oxidizing agent or as a brominating agent and it usually does both when it is allowed to act on camphoroxime. The course to bromonitrocamphane is followed to the larger extent, but some of the hydroxy-nitrosocamphane is almost always present in the final product.

The question as to the correct structural formula of the anhydride formed by the action of conc. sulfuric acid on bromonitro-camphane has not been satisfactorily answered. Empirically, the reaction involves simply the removal of the elements of water to form the anhydride but, as Forster said, owing to the fact that a nitro group is involved in the dehydration, it is difficult to ascribe a structural formula to the product. From the fact $^{3}$ that 2-bromo-1-nitrocamphane as shown below in Formula I does not yield an anhydride, it would seem that both of the hydrogens on that carbon atom are involved in the dehydration. But the bromine atom exerts some influence because ordinary nitrocamphane does not give an anhydride when treated with conc. sulfuric acid. On first thought, it would be expected that Formula II would represent the correct structure of the anhydride.

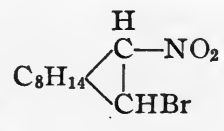

I

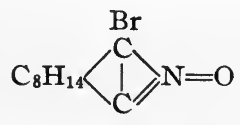

II

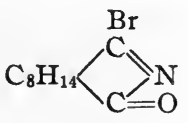

III

It was found, however, by Forster that dil. mineral acids and other milder reagents such as hydroxylamine convert the anhydride into an isomer which gives a benzoyl derivative.

Of course Formula II is not the only formula that can be ascribed to the first anhydride. Very pertinent to this question is the work of Wallach ${ }^{4}$ on the action of sulfuric acid on various oximes. He has shown that in many cases the first step in the reaction is the rearrangement of the oxime to the lactam compound. Following a similar line of reasoning, the oxygen atoms of the nitro group might be thought to rearrange on to the adjacent carbon atom thus forming two hydroxyl groups, and the nitrogen atom might assume a position between the two carbons. Then, of course, the sulfuric acid would remove the elements of water leaving the carbonyl group in the molecule, as in Formula III above. Assuming this to be the structure of the first anhydride, it is probable that the second form of the anhydride, an isomer, could be represented as the enol form as follows.

${ }^{3}$ Forster, J. Chem. Soc., 79, 653 (1901).

${ }^{4}$ Wallach, Ann., 309, 1 (1899); 312, 171 (1900). 


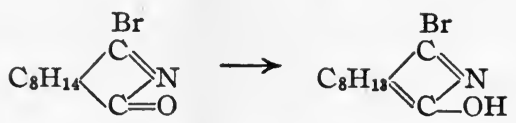

If the carbonyl group is present in the first anhydride, then it should give the regular carbonyl reactions. But Forster, in working on the structure of the anhydride, found that the first isomeric anhydride gave an hydroxylamino compound with hydroxylamine, instead of the oxime which would be the derivative expected. He was unable to cause the carbonyl group to react as such. In carrying out this investigation, recently, it seemed that treatment of the first isomeric anhydride with methyl magnesium iodide in the regular Grignard reaction might shed some light on the structure of the compound. This has been done in this work and it is believed that the results obtained furnish convincing evidence that the structure of the first isomeric anhydride is that which represents it as a substituted inner amide. This was originally proposed by Forster, but he was unable to demonstrate the presence of the carbonyl group, probably because of the fact that the Grignard reaction was not in such general use then as it is at the present time.

When the first isomeric anhydride is treated with methyl magnesium iodide according to the regular Grignard reaction, and the product decomposed with water, a new compound is obtained which has its molecular weight increased by 1 carbon atom and 4 hydrogen atoms. This compound gives a benzoyl derivative by the Schotten-Baumann reaction, and the derivative has one benzoyl group. When the compound produced by the Grignard reaction is treated with alcoholic potash, the bromine atom is lost, the nitrogen atom is eliminated as ammonia, and a methyl ketonic acid obtained whose melting point checks with that acid which would be obtained theoretically in this reaction. ${ }^{5}$ The changes that take place from the first isomeric anhydride would then be represented as follows.

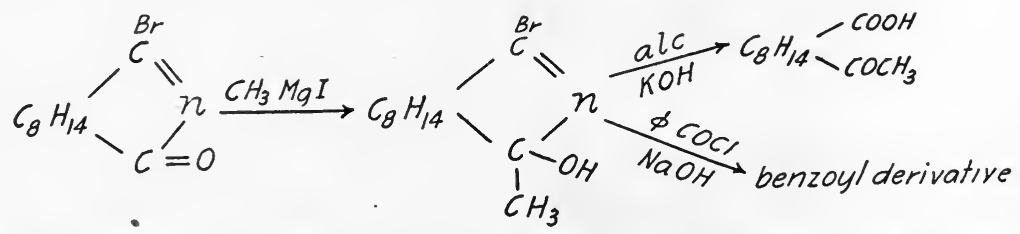

This furnishes even greater confirmatory evidence for the inner substituted amide formula for the first form of the bromonitrocamphane anhydride. In other words, it completes the proof for this structural formula for the first isomer of bromonitrocamphane anhydride and supplements Forster's data for the isomeric compound, the enol form for this same anhydride.

' Winzer, Ann., 257, 311 (1890). 


\section{Experimental}

Preparation of Bromonitrocamphane.-The method employed is essentially the same as used by Forster originally, except that a larger quantity of bromine is added to the reaction mixture. By using a solution of potassium hypobromite in which there is a slight excess of bromine, it has been found that the product comes out almost pure with very little formation of the hydroxy-nitrosocamphane, the green compound. The weights used were $600 \mathrm{~g}$. of bromine, $600 \mathrm{~g}$. of potassium hydroxide, $100 \mathrm{~g}$. of camphoroxime.

Oxidation of Bromonitrocamphane with Nitric Acid.-In this experiment, $25 \mathrm{~g}$. of bromonitrocamphane was oxidized with a nitric acid solution (200 cc. of water to $300 \mathrm{cc}$. of conc. nitric acid). The mixture was refluxed for a week, day and night, and at the end of the time, the camphoronic acid separated as the barium salt of the acid and the camphoric acid as the anhydride. If the same type of oxidation is conducted, except for a shorter time, it is possible to condense considerable quantities of camphor in the condenser tube, showing the successive steps in the oxidation of bromonitrocamphane.

Quantitative Observations on the Course of the Action of Potassium Hypobromite on Camphoroxime.-Working on the theory that, possibly, an intermediate bromonitroso compound might be formed with an appreciable lag in the reaction, and that accordingly there might be a definite variation or break in the curve of the utilization of the potassium hypobromite, the following set of experiments was run to determine whether any sharp variation took place in the action of the potassium hypobromite.

Quantitative amounts of camphoroxime $(6.70 \mathrm{~g}$. or $0.04 \mathrm{~mol}$ ), bromine (10.50 g. or $0.06 \mathrm{~mol}$. ), and potassium hydroxide ( $20 \mathrm{~g}$. in $200 \mathrm{cc}$. of water or $0.35 \mathrm{~mol}$.), were caused to react while the mixture was vigorously stirred. By the removal of aliquot parts of the potassium hypobromite, addition to potassium iodide, and titration of the liberated iodine with standard sodium thiosulfate solution, with starch as an indicator, it was possible to get a relation between the grams of bromine used per unit time. This, plotted, showed that there was no definite break in the curve of the utilization of the bromine.

$\begin{array}{cccccc}\text { Time } & \text { Bromine used } & \text { Time } & \text { Bromine used } & \text { Time } & \text { Bromine used } \\ \text { Hrs. } & \text { G. } & \text { Hrs. } & \text { G. } & \text { Hrs. } & \text { G. } \\ 0.25 & 6.42 & 1.50 & 9.00 & 3.00 & 10.05 \\ 0.50 & 7.27 & 2.00 & 9.35 & 4.00 & 10.48 \\ 1.00 & 8.58 & \ldots & \ldots & \ldots & \ldots\end{array}$

Preparation of Bromonitrocamphane Anhydride (Forster's).-The method given here is modified so that the reaction can be kept under control. Forster originally added the solid bromonitrocamphane directly to the conc. sulfuric acid with the result that, since the reaction is exothermic, considerable decomposition took place and small yields of the anhydride were obtained. His method has been modified by dissolving the bromonitrocamphane in low-boiling petroleum ether, and allowing this to drip slowly, into a cold mixture $\left(-10^{\circ}\right)$ of conc. sulfuric acid and petroleum ether which was stirred vigorously. The bromonitrocamphane is taken over quickly by the sulfuric acid, but when the temperature is kept below $-5^{\circ}$ the yield of the anhydride is almost quantitative and the product is almost pure white, scarcely requiring recrystallization. The anhydride is obtained finally by allowing the conc. acir solution to drip over finely crushed ice, whereby the anhydride is precipitated as a slightly yellow, flocculent solid. This is usually pure enough for most purposes, but it can be recrystallized from hot alcohol to obtain it in pure condition.

Action of the Grignard Reagent (Methyl Magnesium Iodide) on Bromonitrocamphane Anhydride.-Five g. of magnesium turnings is immersed in $200 \mathrm{cc}$. of dry 
ether in a 1-liter flask connected to a reflux condenser with air-tight connections. A small amount of iodine is introduced as a catalyst and $20 \mathrm{~g}$. of methyl iodide is added in portions, so that the vigorous reaction is well under control. The solution is refluxed until all action has ceased and there is only a very small amount of magnesium left in the bottom of the flask. Then $10 \mathrm{~g}$. of the bromonitrocamphane anhydride, dissolved in about $50 \mathrm{cc}$. of dry ether, is added in small portions through the top of the condenser and this mixture refluxed for several hours. The flask is then disconnected, and small amounts of water added cautiously to decompose the Grignard compound. After the main reaction has ceased, some dil. sulfuric acid is added. The whole is stirred well, at least for several minutes and the ether separated by means of a separatory funnel, washed with water, with a solution of sodium carbonate, again with water several times, dried over calcium chloride and evaporated almost to dryness over the steam-bath. At this point, if the liquid residue is allowed to cool slowly so that the last portion of the ether evaporates spontaneously, the derivative crystallizes in large, lustrous, transparent plates, m. p. $117-8^{\circ}$. It is insoluble in acids or alkalies, is soluble in ether or alcohol, but insoluble in water. It is converted readily into the corresponding acyl derivatives.

Analysis. Subs., 0.1705: $\mathrm{AgBr}, 0.1230(\mathrm{Br}, 0.0524)$. Calc. for $\mathrm{C}_{11} \mathrm{H}_{13} \mathrm{ONBr}$; $\mathrm{Br}, 30.75$. Found: 30.73 .

Analysis of the Benzoyl Derivatives of Methyl Bromonitrocamphane Anhydride.By treating the methyl bromonitrocamphane anhydride with benzoyl chloride according to the Schotten-Baumann reaction, a benzoyl derivative is obtained, fine white crystals crystallized from hot dil. alcohol, m. p. 113-114 ${ }^{\circ}$. It will also crystallize from ether in star-shaped needle groups.

Analysis. Subs., 0.2141: $\mathrm{AgBr}, 0.1094$. Calc. for $\mathrm{C}_{18} \mathrm{H}_{22} \mathrm{O}_{2} \mathrm{NBr}$ : $\mathrm{Br}, 21.90$. Found: 21.75 .

Action of Alcoholic Sodium Hydroxide on Methyl Bromonitrocamphane Anhydride. Formation of Aceto-camphenyl Carboxylic Acid.-To observe the action of alcoholic sodium hydroxide on the compound produced by the action of methyl magnesium iodide, which is named methyl bromonitrocamphane anhydride, $5 \mathrm{~g}$. of this derivative was dissolved in $25 \mathrm{cc}$. of ethyl alcohol, $10 \mathrm{cc}$. of conc. sodium hydroxide solution was added and the mixture or solution boiled for a few minutes. A strip of moistened red litmus suspended in the vapors issuing from the flask was turned blue, and the presence of ammonia was also shown by the fumes with hydrochloric acid and the reaction with Nessler solution. The alcohol is boiled off, the residue taken up with ether and water together, acidified, extracted with ether, the acids extracted from the ether with a solution of sodium hydroxide, and the acids again liberated from the alkali by acid and removed to the ether layer. The ether, evaporated spontaneously, left slightly yellowish crusts, which are soluble in alkali, but insoluble in water. A sample of these crusts when tested according to the iodoform reaction gave a heavy precipitate of iodoform; m. p., $67-70^{\circ}$. The wide range of temperature was probably due to slight impurities, as the quantity was too small to purify as much as desired. The compound gave negative qualitative tests for halogen and nitrogen. This substance should be the methyl ketonic acid, aceto-camphenyl-carboxylic acid prepared by Winzer, ${ }^{6}$ if the structures of the preceding compounds are as stated.

\section{Summary}

1. Bromonitrocamphane on oxidation with nitric acid breaks down successively into camphor, camphoric acid and camphoronic acid.

2. The course of the action of potassium hypobromite on camphoroxime has been found to be bromination of the camphoroxime and then oxida- 
tion to the bromonitrocamphane. No bromonitrocamphane hydrate is formed, as was supposed by Forster.

3. Potassium hypobromite may also act as an oxidizing agent on camphoroxime to produce hydroxy-nitrosocamphane.

4. Bromonitrocamphane anhydride has been prepared by an improved method and the structure of the anhydride elucidated by its behavior with the Grignard reagent, methyl magnesium iodide. Since the structure of the anhydride is now an established fact, a logical explanation is provided by the unexpected transition from bromonitrocamphane to infra-campholenenitrile.

5. In general, this work fits in with and substantiates the work of Forster on these compounds. 


\section{VI'TA}

The author was born on the 7th day of April, 1897, at Columbia, Missouri. He attended several grade schools, finishing the eighth grade at Macomb, Illinois. Then he entered the Western Illinois State Teachers' College Academy and graduated from there at the end of four years, 1911-15. In the fall of the same year, he entered the University of Illinois in the regular chemistry course, and graduated with the B.S. degree in June, 1919. Graduate work was begun in the same University and the M.S. degree received in June, 1920. From that time to the present, the author has been doing further graduate work here.

The writer taught quantitative chemistry as Graduate Assistant during the year, 1920-21, in the University of Illinois.

Publications:- "Syntheses of Chromanes and Coumaranes" Journal of the American Chemical Soc., Vol. 42, 157 (1920), by R. E. Rindfusz, P. M. Ginnings, V. L. Harnack.

Fraternities and Societies:-Gammi Pi Upsilon, Phi Lambda Upsilon, Associate Member of Sigma Xi, and American Chemical Society. 




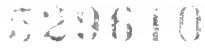

UNIVERSITY OF CALIFORNIA LIBRARY 


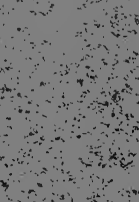

and

4

$a_{-2}$

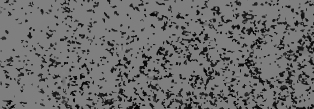

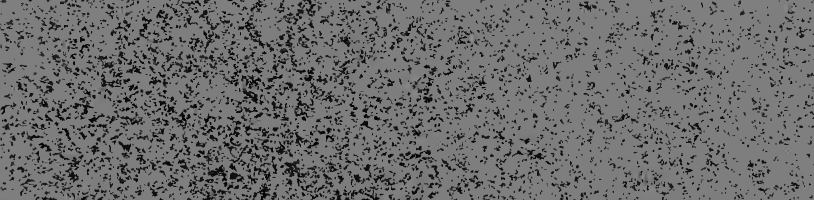

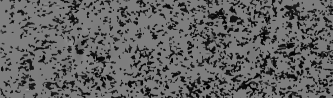

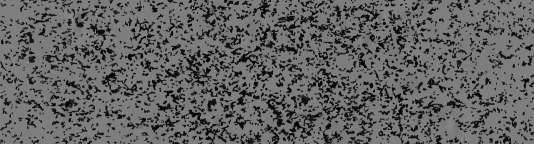
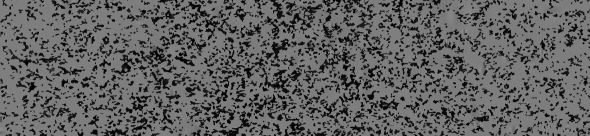

ins $x+3 y$

nom

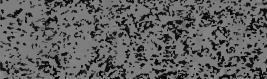

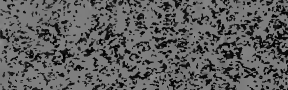

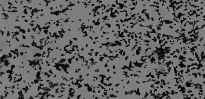

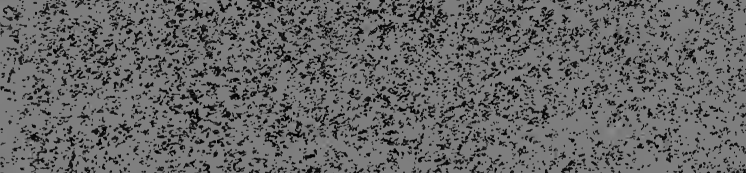

